\title{
Biochemical Alterations in Diabetic Neuropathy
}

Subhashree Venugopal ${ }^{*}$

VIT University, Tamil Nadu, India

*Corresponding author: Subhashree Venugopal, M.Sc., Ph.D, VIT University, Tamil Nadu, India, E-mail: v_subhashree@hotmail.com

Received date: May 20, 2014, Accepted date: November 17, 2014, Published date: November 24, 2014

Copyright: (c) 2014 Venugopal S. This is an open-access article distributed under the terms of the Creative Commons Attribution License, which permits unrestricted use, distribution, and reproduction in any medium, provided the original author(s) and source are credited.

\begin{abstract}
Type 2 Diabetes mellitus is a disease which manifests with a variety of cardiovascular risk factors, including hypertension, dyslipidemia and overweight or obesity that contributes to development of long term complications termed diabetic related ailments. Diabetic peripheral neuropathy (DPN) is a debilitating condition affecting as many as one half of all patients with diabetes during the course of their disease. Several metabolic and vascular pathways have been identified as contributors to the pathogenesis of diabetic neuropathy. Diabetic neuropathy encompasses a wide range of clinical and subclinical syndromes from pain to complete loss of sensation. Metabolic and therapeutic approaches have focused on aldose reductase, poly (ADP- Ribose) polymerase, protein kinase C, advanced Glycation end products. Novel approaches to identify targets for treatment of diabetic peripheral neuropathy require cross links between molecular and computational biology methods.
\end{abstract}

Keywords: Peripheral neuropathy; Aldose reductase; Glyoxalase; Protein kinase C; AGEs; PARP

\section{Introduction}

Diabetes mellitus is one of the most common non-communicable diseases worldwide. It is the fourth or the fifth leading cause of death in most developed countries, and substantial evidence points to an epidemic of diabetic mellitus in many developing and newly industrialized nations [1].The rapid rise in its prevalence has been driven over recent decades by changes in environment including lifestyle of populations [2]. Chronic hyperglycemia is a wellestablished cause of micro vascular complications [3]. Peripheral neuropathy is a common complication in patients with diabetes mellitus [4]. Diabetic peripheral neuropathy affects at least $50 \%$ of patients with type 1 and type 2 diabetes [5]. Biochemical changes found in diabetic neuropathy are more wide spread and more controversial than anatomic changes [6]. Different hypothesis for the pathogenesis of diabetic complications besides increased polyol pathway activity have been proposed including altered protein kinase $\mathrm{C}$ activity, increased oxidative stress and an acceleration of nonenzymatic glycation [7]. Haimanot and Abdulkadir [8] also suggested a relation between diabetic neuropathy and duration of diabetes, but no relation between neuropathy and the age of patients. Therefore, $10 \%$ of the diabetic population diagnosed for less than one year suffers of neuropathy while this number increases to up to $50 \%$ of the diabetic population diagnosed for more than 25 years. It is generally accepted that $30 \%$ of the diabetic population suffers from diabetic neuropathy.

\section{Pathogenesis and clinical indications of diabetic peripheral neuropathy}

Diabetic peripheral polyneuropathy (DPN) is a multifactorial disorder arising from hyperglycemia and or insulin deficiency. Two main hypotheses have been proposed for the pathogenesis of diabetic neuropathy although it is not clearly established. One is the metabolic derangement theory, hyperglycemic activation of the polyol pathway leading to accumulation of sorbitol and potential changes in theNAD: $\mathrm{NADH}$ ratio may cause direct neuronal damage and/or decreased nerve blood flow [9]. The other is that endoneurial micro vascular lesions which plays an important role in diabetic neuropathy. At present, it is thought that both metabolic and vascular factors play roles in the pathogenesis of clinical neuropathy. The pathophysiological mechanisms of diabetic neuropathy (DN) are complex and involve the activation of numerous pathways involving nerve growth factors, inflammatory mediators, and reactive oxygen and nitrogen species [10]. Advanced DPN causes serious complications such as diabetic foot ulcers, gangrene and charcot joint, all of which reduce the quality of life of patients with DPN [11]. DPN is often characterized by damage to both large as well as small thinly myelinated C fibers. Small fiber DPN is associated with increased morbidity and mortality [12]. Symptoms include numbness, pain and decreased sensation as well as autonomic symptoms such as anhidrotic skin, orthostatic hypotension, resting tachycardia, hypoglycemia unawareness, delayed gastric emptying, decreased bladder tone and impotence [12].

\section{Biochemical Factors and Diabetic Neuropathy}

\section{$\mathrm{Na}+/ \mathrm{K}+\mathrm{ATPase}$ activity}

The factors include decreased $\mathrm{Na}^{+} / \mathrm{K}^{+}$ATPase activity, increased anaerobic glycolysis, increased myoinositol, nerve ischemia due to microangiopathy [13]. From biochemical studies in rat diabetic models, it is known that decreased $\mathrm{Na}^{+} / \mathrm{K}^{+}$ATPase activity in diabetic subjects reverses rapidly after normalization of blood glucose levels [14]. There is a reduction in catecholaminergic neuronal ability with a reduction in catecholamine synthesis associated with a difficulty to release the neurotransmitters from synaptic terminals [6]. The study reported that alterations in catecholamine metabolism are dependent on the severity and duration of diabetes. Short term diabetes is accompanied with increase or no change in catecholamine content or release, but reduced release and increased storage of catecholamines are found in long term diabetes [15]. 
Page 2 of 4

\section{Advanced glycation end products}

The enhanced formation of advanced glycation end products (AGEs) induced by hyperglycemia has been implicated in pathogenesis of diabetic complications [16]. The oxidative stress may accelerate auto oxidation of glucose to dicarboxyl compounds (glyoxal) that are precursors of $\mathrm{N}-\varepsilon$ (carboxymethyllysine) (CML) [17] and glycooxidation of amadori products to CML [18]. The main source of reactive oxygen species in diabetes is thought to be mitochondria mediated or mitochondria dependent [19].

\section{Uncoupled proteins}

Uncoupled proteins (UCPs) can provide a controlled leak of protons across the inner membrane of mitochondria, thus uncouple oxidative phosphorylation from respiration with a concomitant decrease in mitochondrial membrane potential [20] and free radical generation [21]. UCP2 which is expressed in various human tissues, is thought to control body temperature, energy metabolism as well as to regulation of mitochondrial production of ROS (Reactive Oxygen species). Hence, UCP2 gene is considered to be involved in DPN. A study revealed that the $-866 \mathrm{G} / \mathrm{A}$ and A/A genotypes of UCP2 are significantly associated with nerve conduction slowing and impaired blood pressure regulation on a head-up tilt test. This suggests that higher UCP2 activity related to the A allele of - 866 G/A polymorphism that causes deterioration of peripheral nerve function by energy depletion rather than neuroprotective effect against oxidative stress in type 2 diabetic patients [22].

\section{Polyol pathway}

Elevated blood glucose in diabetic patients leads to increased activity of aldose reductase, anenzyme that converts glucose to sorbitol, one of the alcohol sugars. The result is accumulation of sorbitol within nerves, which is associated with oxidative stress and nerve damage. The polyol pathway secondarily converts sorbitol into fructose .The polyol pathway plays an important role in the development and progression of diabetic neuropathy. Enhanced catabolism of glucose via polyol pathway is known to augment reactive oxygen species by mechanisms such as glutathione depletion or increased prostaglandin synthesis [23]. Superoxide dismutase is known to lose its activity as a consequence of reacting with reducing sugars or other intermediate metabolites which in turn augments radical species [24]. Diabetes is one of the diseases that activates ubiquitin proteasome pathway and it has been proposed that activation of this pathway is responsible for wasting muscle because of insulopenia. The activation of ubiquitin proteasome pathway in some neuronal diseases and insulopenia suggests that ubiquitin may play a role in diabetic neuropathy [25].

Oxidative nitrosative stress manifest by accumulation of lipid peroxidation products, 4 hydroxynonal adducts nitrated proteins and 8 -hydroxy guanosine in peripheral nerve, spinal cord and dorsal root ganglion neurons of diabetic rats or mice [26]. Oxidative nitrosative stress is a key mechanism contributing to nerve blood flow and nerve conduction deficits, small sensory nerve fibre dysfunction and morphological manifestation of diabetic peripheral polyneuropathy.

\section{Metanx}

Metanx containing 1-methyl folate, pyridoxal 5' phosphate and methylcobalamine was evaluated on oxidative nitrosative stress and manifestations of DPN in Zucker diabetic fatty acid rats. The high efficacy of metanx is probably explained by its interactions of its components counteracting oxidative nitrosative stress through restoration of eNOS (endothelial nitric oxide synthase) coupling in vasa nervorum. (L-methylfolate), neutralization of superoxide and peroxynitrite (methylcobalamine) and chelation of transition metals and abrogation of advanced glycation end products formation. (Pyridoxal 5' phosphate) [27]. Metanx alleviated sensory neuropathy without affecting MNCV (motor nerve conduction velocity) or morphometric characteristics of large myelinated fibers [27]. Fonsceva et al. [28] conducted a multicenter, randomized, double blind placebo trial consisting of 214 patients for 24 weeks to assess whether Metanx would improve objective measures of diabetic peripheral neuropathy. The study provides evidence that Metanx is a safe and effective therapy for alleviation of peripheral neuropathy symptoms, at least in short term.

\section{Glutathione}

The administration of reduced glutathione has partially improved experimental diabetic neuropathy [29]. Experimental diabetic neuropathy results in a reduction in nerve blood flow(NBF) by $50 \%$ nerve conduction slowing and a large decrease in glutathione and that lipoic acid supplementation resulted in a dose dependent normalization of NBF and glutathione suggest that neuropathy is due in significant part due to oxidative stress and that improving free radical scavenging capacity is responsible for improvement in NBF and neuropathy and a time and dose dependent improvement in digital nerve conduction velocity [30]. One mechanism of reduced NBF is inhibitory effect of superoxide anion on nitric oxide synthase with resultant decreased NO in Experimental neuropathy [31].

\section{Alpha lipoic acid}

Alpha lipoic acid which has been shown to be effective in both somatic and autonomic neuropathies in diabetes, normalizes endoneural blood flow [30] reduces oxidative stress [32] and improves vascular dysfunction [33]. In a placebo controlled trial in patients with diabetic neuropathy, a significant relief of neuropathic symptoms was observed in patients who received alpha lipoic acid [34].

A meta-analysis that combined the results of four randomized trials, including 1258 patients, found that treatment with $600 \mathrm{mg} /$ day of intravenous racemic lipoic acid for 3 weeks significantly reduced the symptoms of diabetic neuropathy to a clinically meaningful degree [35].

\section{Acetyl carnitine}

Acetyl carnitine is deficient in diabetic condition. In preclinical studies, substitution with acetyl carnitine corrects perturbation of neural $\mathrm{Na}+\mathrm{K}+$ ATPase, myoinositol, NO, prostaglandin and lipid peroxidation all of which play important early pathogenic roles in diabetic peripheral neuropathy [36].

In a 52 week randomized placebo controlled study of 1257 patients with diabetic neuropathy 2 doses of acetyl carnitine, 500 and 1000 $\mathrm{mg}$ /day t.i.d (three times a day) were tested and results demonstrated significant improvement in pain and vibration perception association with improvements in sural nerve morphometry in patients treated with $1000 \mathrm{mg}$ acetyl carnitine t.i.d for one year [37]. 
Page 3 of 4

\section{Resveratrol}

Resveratrol was demonstrated to induce effects that may contribute to the protection of $\beta$ cells in diabetes. In experiments on pancreatic islets, the ability of resveratrol to reduce insulin secretion was demonstrated; this effect was confirmed in animals with hyperinsulinemia, in which resveratrol decreased blood insulin levels [38]. Using a randomized double-blind placebo-controlled clinical trial, a study examined the effects of resveratrol in lowering blood glucose and other related outcomes (e.g., insulin, metabolic markers, cardiovascular risk factors) in patients with type 2 diabetes. In the randomized, double-blind, placebo-controlled clinical trial study, intake of $1 \mathrm{~g} / \mathrm{d}$ of resveratrol for 45 days was found to significantly reduce systolic blood pressure, fasting blood glucose, hemoglobin A1c, insulin, and insulin resistance, while HDL (high density lipoprotein) was significantly increased, when compared to their baseline levels [39].

\section{Glyoxalase 1}

Glyoxalase 1 (Glo1) and glyoxalase 2 (Glo2) present an enzymatic defense system against glycation that suppresses glycation mediated cell damage [40]. Altered Glo1 activity is associated with late diabetic complications [41]. Increased formation of methylglyoxal in diabetesassociated hyperglycaemia leads to a $2-4$ fold increase in modifications of proteins by methylglyoxal to form AGEs at the sites of vascular complications [42]. Glo1 activity was significantly reduced in patients with severe painful neuropathy symptoms for both type 1 and type 2 diabetes mellitus patients. The molecular mechanisms that are linked to the altered activity of Glo1 deserve further investigations. The pathogenesis of type 2 diabetes mellitus is considerably heterogeneous and both glycation stress and inflammation-related processes could be cooperatively driving forces in the development of late complications of diabetes mellitus [43].

\section{Poly (ADP- Ribose) polymerase}

In Diabetic neuropathy, elevated glucose level increases the ROS production and these free radicals induce DNA strand breaks, thereby activating PARP. After sensing the DNA damage, PARP gets activated and repairs the DNA by transferring ADP-ribose unit to the nuclear proteins and depletes the intra-cellular NAD. Intra-cellular NAD resynthesis consumes ATP which further leads to impairment of several NAD dependent pathways like glycolysisand mitochondrial respiration. The energy consuming cycle results in rapid depletion of intra-cellular $\mathrm{NAD}^{+}$and ATP pools and ultimately the cell dysfunction [44]. PARP over activation has shown increased sensitivity to mechanical noxious stimuli associated with diabetes [45].

\section{Protein Kinase C}

Protein kinase $\mathrm{C}$ is overactivated by hyperglycemia and by disordered fatty acid metabolism resulting in increased production of vasoconstrictive, angiogenic and chemotactic cytokines including transforming growth factor, vascular endothelial growth factor inhibitors. Protein kinase $\mathrm{C}$ overactivation blocking is a critical step in the pathogenesis of diabetic polyneuropathy via its impact on microvascular mechanism [46].

A number of aldose reductase inhibitors have been developed [47]. But none have achieved success for diverse reasons, one being that not all aldose reductase inhibitors penetrate human peripheral nerves. A paradigm shift is imminent in the research arena of diabetic neuropathy using molecular and bioinformatics approaches to reduce the pain and symptoms experienced in late stages of type 2 diabetes. Multiple biochemical pathways have been implicated in the pathogenesis of diabetic peripheral neuropathy. It has been demonstrated that strict glycemic control alone can decrease the incidence of diabetic complications [48]. However, it is impossible to completely prevent the development of diabetic complications solely by glycemic control. The future therapeutic approach which needs to be reckoned with, must focus on developing novel drugs with better efficacy that elicit better response in patients. Moreover, it is imperative to target critical cellular pathways leading to increased oxidative stress production and thereby to enhance the antioxidant defense potential to mitigate diabetic peripheral neuropathy state.

\section{References}

1. Zimmet P, Alberti KG, Shaw J (2001) Global and societal implications of the diabetes epidemic. Nature 414: 782-787.

2. Wild S, Roglic G, Green A, Sicree R, King H (2004) Global prevalence of diabetes: estimates for the year 2000 and projections for 2030. Diabetes Care 27: 1047-1053.

3. (1993) The effect of intensive treatment of diabetes on the development and progression of long-term complications in insulin-dependent diabetes mellitus. The Diabetes Control and Complications Trial Research Group. N Engl J Med 329: 977-986.

4. Low PA (1987) Recent advances in the pathogenesis of diabetic neuropathy. Muscle Nerve 10: 121-128.

5. Boulton AJ, Vinik AI, Arezzo JC, Bril V, Feldman EL, et al. (2005) Diabetic neuropathies: a statement by the American Diabetes Association. Diabetes Care 28: 956-962.

6. Gallego M, Setién R, Izquierdo MJ, Casis O, Casis E (2003) Diabetesinduced biochemical changes in central and peripheral catecholaminergic systems. Physiol Res 52: 735-741.

7. Greene DA, Sima AA, Stevens MJ, Feldman EL, Lattimer SA (1992) Complications: neuropathy, pathogenetic considerations. Diabetes Care 15: 1902-1925.

8. Haimanot RT, Abdulkadir J (1985) Neuropathy in Ethiopian diabetics: a correlation of clinical and nerve conduction studies. Trop Geogr Med 37: 62-68.

9. Greene DA, Lattimer SA (1983) Impaired rat sciatic nerve sodiumpotassium adenosine triphosphatase in acute streptozocin diabetes and its correction by dietary myo-inositol supplementation. J Clin Invest 72: 1058-1063.

10. Negi G, Kumar A, Sharma SS (2010) Concurrent targeting of nitrosative stress-PARP pathway corrects functional, behavioral and biochemical deficits in experimental diabetic neuropathy. Biochem Biophys Res Commun 391: 102-106.

11. Jude EB, Boulton AJM (1999) End-stage complications of diabetic neuropathy. Diabetes Rev 7: 395-410.

12. Lacomis D (2002) Small-fiber neuropathy. Muscle Nerve 26: 173-188.

13. Schneider U, Niedermeier W, Grafe P (1993) The paradox between resistance to hypoxia and liability to hypoxic damage in hyperglycemic peripheral nerves. Evidence for glycolysis involvement. Diabetes 42: 981-987.

14. Raccah D, Fabreguetts C, Azulay JP, Vague P (1996) Erythrocyte Na(+)$\mathrm{K}(+)$-ATPase activity, metabolic control, and neuropathy in IDDM patients. Diabetes Care 19: 564-568.

15. Gallego M, Casis O (2001) Regulation of cardiac transient outward potassium current by norepinephrine in normal and diabetic rats. Diabetes Metab Res Rev 17: 304-309.

16. Brownlee M (1995) Advanced protein glycosylation in diabetes and aging. Annu Rev Med 46: 223-234.

17. Wells-Knecht KJ, Zyzak DV, Litchfield JE, Thorpe SR, Baynes JW (1995) Mechanism of autoxidative glycosylation: identification of glyoxal and 
arabinose as intermediates in the autoxidative modification of proteins by glucose. Biochemistry 34: 3702-3709.

18. Nagai R, Ikeda K, Higashi T, Sano H, Jinnouchi Y, et al. (1997) Hydroxyl radical mediates $\mathrm{N}$ epsilon-(carboxymethyl)lysine formation from Amadori product. Biochem Biophys Res Commun 234: 167-172.

19. Brownlee $\mathrm{M}$ (2001) Biochemistry and molecular cell biology of diabetic complications. Nature 414: 813-820.

20. Bouillaud F, Ricquier D, Thibault J, Weissenbach J (1985) Molecular approach to thermogenesis in brown adipose tissue: cDNA cloning of the mitochondrial uncoupling protein. Proc Natl Acad Sci U S A 82: 445-448.

21. Arsenijevic D, Onuma H, Pecqueur C, Raimbault S, Manning BS, et al. (2000) Disruption of the uncoupling protein-2 gene in mice reveals a role in immunity and reactive oxygen species production. Nat Genet 26: 435-439.

22. Yamasaki H , Sasaki H, Ogawa K, Shono T, Tamura S, et al. (2006) Uncoupling Protein 2 Promoter Polymorphism -866G/A Affects Peripheral Nerve Dysfunction in Japanese Type 2 Diabetic Patients. Diabetes Care 29: 888-895.

23. Kashiwagi A, Asahina T, Ikebuchi M, Tanaka Y, Takagi Y, et al. (1994) Abnormal glutathione metabolism and increased cytotoxicity caused by $\mathrm{H} 2 \mathrm{O} 2$ in human umbilical vein endothelial cells cultured in high glucose medium. Diabetologia 37: 264-269.

24. Ookawara T, Kawamura N, Kitagawa Y, Taniguchi N (1992) Site-specific and random fragmentation of $\mathrm{Cu}, \mathrm{Zn}$-superoxide dismutase by glycation reaction. Implication of reactive oxygen species. J Biol Chem 267: 18505-18510.

25. Price SR, Bailey JL, Wang X, Jurkovitz C, England BK, et al. (1996) Muscle wasting in insulinopenic rats results from activation of the ATPdependent, ubiquitin-proteasome proteolytic pathway by amechanism including gene transcription. J Clin Invest 98: 1703-1708.

26. Obrosova IG (2009) Diabetes and the peripheral nerve. Biochim Biophys Acta 1792: 931-940.

27. Shevalye H, Watcho P, Stavniichuk R, Dyukova E, Lupachyk S, et al. (2012) Metanx alleviates multiple manifestations of peripheral neuropathy and increases intraepidermal nerve fiber density in Zucker diabetic fatty rats. Diabetes 61: 2126-2133.

28. Fonseca VA, Lavery LA, Thethi TK, Daoud Y, DeSouza C, et al. (2013) Metanx in type 2 diabetes with peripheral neuropathy: a randomized trial. Am J Med 126: 141-149.

29. Bravenboer B, Kappelle AC, Hamers FP, van Buren T, Erkelens DW, et al. (1992) Potential use of glutathione for the prevention and treatment of diabetic neuropathy in the streptozotocin-induced diabetic rat. Diabetologia 35: 813-817.

30. Nagamaatsu M, Nickander KK, Schmelzer JD, Raya A, Wittrock DA, et al. (1995) Lipoic acid improves nerve blood flow, reduces oxidative stress and improves distal nerve conduction in Experimental Diabetic neuropathy, Diabetes Care 18: 1160-67.

31. Kihara M, Low PA (1994) Impaired vasoreactivity to nitric oxide and endothelin vasoreactivity in experimental diabetic neuropathy(Abstract). Neurology 44:A274.

32. Low PA, Nickander KK, Tritschler HJ (1997) The roles of oxidative stress and antioxidant treatment in experimental diabetic neuropathy. Diabetes 46 Suppl 2: S38-42.
33. Morcos M, Borcea V, Isermann B, Gehrke S, Ehret T, et al. (2001) Effect of alpha-lipoic acid on the progression of endothelial cell damage and albuminuria in patients with diabetes mellitus: an exploratory study. Diabetes Res Clin Pract 52: 175-183.

34. Ziegler D, Hanefeld M, Ruhnau KJ, Hasche H, Lobisch M, et al. (1999) Treatment of symptomatic diabetic polyneuropathy with the antioxidant alpha-lipoic acid: a 7-month multicenter randomized controlled trial (ALADIN III Study). ALADIN III Study Group. Alpha-Lipoic Acid in Diabetic Neuropathy. Diabetes Care 22: 1296-1301.

35. Ziegler D, Nowak H, Kempler P, Vargha P, Low PA (2004) Treatment of symptomatic diabetic polyneuropathy with the antioxidant alpha-lipoic acid: a meta-analysis. Diabet Med 21: 114-121.

36. Scarpini E, Doneda P, Pizzul S, Chiodi P, Ramacci MT, et al. (1996) Lcarnitine and acetyl-L-carnitine in human nerves from normal and diabetic subjects. J Peripher Nerv Syst 1: 157-163.

37. SimaAA, Calvani M, Mehra M, Amato A (2005) Acetyl-L-Carnitine Study Group: Acetyl-L-carnitineimproves pain, nerve regeneration, and vibratory perception in patients with chronic diabeticneuropathy: an analysis of two randomized placebo-controlled trials. Diabetes Care 28: 89-94.

38. Szkudelski T, Szkudelska K (2011) Anti-diabetic effects of resveratrol. Ann N Y Acad Sci 1215: 34-39.

39. Movahed A, Nabipour I, Lieben Louis X, Thandapilly SJ, Yu L, et al. (2013) Antihyperglycemic effects of short term resveratrol supplementation in type 2 diabetic patients. Evid Based Complement Alternat Med 2013: 851267.

40. Thornalley PJ (2008) Protein and nucleotide damage by glyoxal and methylglyoxal in physiological systems--role in ageing and disease. Drug Metabol Drug Interact 23: 125-150.

41. Thornalley PJ, Hooper NI, Jennings PE, Florkowski CM, Jones AF, et al. (1989) The human red blood cell glyoxalase system in diabetes mellitus. Diabetes Res Clin Pract 7: 115-120.

42. Rabbani N, Thornalley PJ (2012) Methylglyoxal, glyoxalase 1 and the dicarbonyl proteome. Amino Acids 42: 1133-1142.

43. Skapare E, Konrade I, Liepinsh E, Strele I, Makrecka M, et al. (2013) Association of reduced glyoxalase 1 activity and painful peripheral diabetic neuropathy in type 1 and 2 diabetes mellitus patients. Journal of Diabetes and Its Complications 27: 262-267.

44. Pacher P, Obrosova IG, Mabley JG, Szabó C (2005) Role of nitrosative stress and peroxynitrite in the pathogenesis of diabetic complications. Emerging new therapeutical strategies. Curr Med Chem 12: 267-275.

45. Ilnytska O, Lyzogubov VV, Stevens MJ, Drel VR, Mashtalir N, et al. ( 2006) Poly(ADP-ribose) polymerase inhibition alleviates experimental diabetic sensory neuropathy. Diabetes 55: 1686-1694.

46. Way KJ, Katai N, King GL (2001) Protein kinase C and the development of diabetic vascular complications. Diabet Med 18: 945-959.

47. Pfeifer MA, Schumer MP, Gelber DA (1997) Aldose reductase inhibitors: the end of an era or the need for different trial designs? Diabetes 46 Suppl 2: S82-89.

48. (1998) The effect of intensive diabetes therapy on measures of autonomic nervous system function in the Diabetes Control and Complications Trial (DCCT). Diabetologia 41: 416-423. 